\title{
An Online Poultry Diseases Monitoring System for Nigeria
}

\author{
Moses Oyekunle Oyetunji ${ }^{1}$, Florence Alaba Oladeji ${ }^{2}$, Olorunjube James Falana ${ }^{1}$, \\ Peter Adebayo Idowu ${ }^{3}$ \\ ${ }^{1}$ Department of Computer Science and Mathematics, Mountain Top University, Ibafo, Nigeria \\ ${ }^{2}$ Department of Computer Science, University of Lagos, Akoka, Lagos, Nigeria \\ ${ }^{3}$ Department of Computer Science and Engineering, Obafemi Awolowo University, Ile-Ife, Nigeria \\ Email address: \\ mooyetunji@mtu.edu.ng (M. O. Oyetunji), foladeji@unilag.edu.ng (F. A. Oladeji), paidowu@oauife.edu.ng (P. A. Idowu)
}

\section{To cite this article:}

Moses Oyekunle Oyetunji, Florence Alaba Oladeji, Olorunjube James Falana, Peter Adebayo Idowu. An Online Poultry Diseases Monitoring System for Nigeria. American Journal of Software Engineering and Applications. Vol. 6, No. 2, 2017, pp. 18-28. doi: 10.11648/j.ajsea.20170602.12

Received: March 21,2017; Accepted: March 31,2017; Published: April 24, 2017

\begin{abstract}
Poultry disease statistics in Nigeria reveal a serious and growing problem with absolute fatality rate and casualty figure rising rapidly. A crucial goal of infectious disease surveillance is the early detection of epidemics, which is essential for disease control. The current system for monitoring poultry disease is based on conventional method using extension workers and confirmed case reports which has proved ineffective over the years. The poultry disease monitoring system aimed at assisting poultry farmers, healthcare officers and stakeholders alike to understand the distribution of poultry diseases and the state of poultry farms in Nigeria in order to ensure early detection and response during an outbreak. The system requirement was elicited from poultry farmers located within the south-western part of Nigeria using interviews while the requirements were designed using use-case diagrams for the representing data insertion and data query. The system was implemented using HTML, PHP and CSS while the database was implemented using MYSQL. In conclusion, the use of monitoring system would be of great importance in providing accurate, timely and detailed information about poultry disease in Nigeria. It will also help to alert disease control agencies about poultry related diseases in Nigeria.
\end{abstract}

Keywords: Poultry Disease, Monitoring System, Use-Case Modeling, Web 2.0, Software

\section{Introduction}

Poultry is an important element for the diversification of Nigeria's economy and increasing household food security [1]. The importance of poultry in national economies of developing countries and its role in improving the nutritional status and incomes of many small farmers and landless peasants has been recognized by various scholars and rural development agencies [2].

Poultry outnumbers all other forms of livestock in Nigeria and are found in most part of the country and chickens remain the most common poultry birds reared across Nigeria's poultry farms (Adene and Oguntade, 2006). Most of these chickens are maintained under traditional, low input, free-range systems of management however, substantial numbers are also reared intensively on a commercial basis (Adene and Oguntade, 2006). No doubt, Chickens provide readily harvestable animal protein to rural/urban households, and in some parts of Africa they are also raised to meet the obligations of hospitality to honored guests. Estimates of livestock population in the last two decades before the outbreak of Avian Influenza in Nigeria shows that poultry industry is the highest and fastest growing livestock subsectors [3]. Unfortunately, the major constraint on traditional or commercial chicken production in Nigeria are poultry diseases especially the Newcastle disease that particularly affects local breeds during certain season of the year [4-6].

Poultry diseases are diseases that can affect the health, production and the poultry population in a given poultry farm or country; some of the commonest diseases that affect poultry are: Newcastle (ND), fowl pox, coccidiosis, external and internal parasites, chronic respiratory diseases (CRD), mycoplasmosis, pullorum, tuberculosis, pneumonia, dysentery, typhoid, aspergillosis and nutritional deficiencies [7].

Flock diseases have been the bane of profitable poultry 
production, particularly in rural areas with little or no access to effective drugs and vaccines. Newcastle Disease (ND) is by far the most predominant infection in both commercial and local chickens in Nigeria [6]. Most public poultry diseases are as a result of environmental problems such as poor sanitary condition, overpopulation of chicks, insufficient or contaminated water and unhygienic disposal of waste.

Poultry diseases have highly destructive effects around the world and these diseases have affected both human and birds. While the developed nations are taking adequate steps to address the poultry related problems, most developing nations are yet to understand the magnitude of the impact of the problem. Poultry related diseases such as Avian Influenza (Bird flu) and Newcastle disease are contagious and can constitute a life threatening menace to both human and birds [3]. Poultry diseases are threat to the growth and survival of the poultry industry. The diseases of chickens need to be observed intensively because of its impact on the health and quality of chickens as poor monitoring system will reduce the productivity and increase its mortality rate.

The use of computers, projectors, magnetic board and CDs has become in vogue in teaching and research. Lecturers routinely use the United States Department of Agriculture USDA/APHIS Animal Health Emergency Management Training CD on Foreign Animal Diseases and the AU-IBAR Community Animal Health Workers Scheme CD to teach at undergraduate and postgraduate levels. The materials have also been used in extension education of community-based rural livestock producers. Veterinary students are also routinely referred to varieties of online website to access the gamut of relevant veterinary education info rmation [8].

ICTs are important to poultry farming and research. Their impacts and challenges are significant; as is their role in providing improved acquisition of skills and competences in poultry production fields, food animal management, food safety, public health, bioinformatics, genetic research, including many other areas such as genomic selection and animal breeding based on thousands of single nucleotide polymorphisms [9]. This was made possible through the Internet and the use of digital devices for data storage, computation and communication, ancillary digital devices connected to computers, personal digital assistants and servers for teaching and learning purposes in both public and private analytical laboratories and veterinary hospitals. Other than laboratory and clinical diagnosis of diseases in domestic, exotic and wild animals or hospital-organizational models, ICTs are also responsible for new veterinary paradigms that are improved by geospatial information technologies. To prevent economic losses due to diseases, poultry farmers should be able to recognize them early and take corrective measures or seek expert advice on what to do.

Monitoring system can be used for disease surveillance, tracking, checking, and continuous record keeping. Poultry diseases are contagious and can easily spread to birds and human. Epidemic diseases have highly destructive effects around the world and these diseases have affected both developed and developing nations [10].

This justifies the prompt need for monitoring system to monitor and give early warning about an outbreak across the globe especially in sub-Sahara Africa (SSA). Monitoring systems are currently been used by government and private organizations to obtain relevant data, access situations and to respond quickly during emergencies. The timely transmission of surveillance data is critical for the development of surveillance or monitoring system, the widespread application of modern telecommunication network and technology make the rapid electronic data entry, reporting and analysis possible in resource-limited areas [11].

In remote areas without computers, mobile phones are commonly used among residents, which can be used for data reporting and transfer. For example a research conducted in 2005 revealed that adopters, potential adopters, and nonadopters of mobile phones represented 59.6\%, 18.8\% and $21.6 \%$ of the rural residents in Hubei province, a middle- and low- income province in China (Wei et al., 2008). In 2008, when the earthquake occurred in Sichuan which caused CISDCP paralyzed for several days, a cellular phone reporting program was initiated emergently by China CDC for infectious disease reporting $[12,13]$.

In the recent past, the use of information and communication technology for farm monitoring system are not widely practiced as most developing countries often depends on the use of extension workers for disease data and monitoring. Several published literature discussed the used of information and communication technology for monitoring cattle, horses, sheep and pigs [1] while monitoring system in poultry farm often focuses on its environment condition such as cage sanitation, air in the cage as well as particles in the air in the cage [7].

Many developed countries are using web based disease surveillance system for the early detection and response to disease of public health importance, especially United States [14]. It is reported there were more than 100 different US health jurisdictions using disease surveillance system to augment their public health surveillance. In Asian countries, Japan, Korea and Taiwan also developed disease surveillance systems for disease outbreak detection [15].

In Nigeria, ICT-based applications dedicated to veterinary medicine, including websites and online resources are becoming increasingly prevalent. Education resources in this field of practice are actively being promoted in Nigeria, and include very comprehensive articles on the application of ICT to veterinary medicine [16]; Simões, 2010.

In Nigeria, the component of the EU-sponsored PanAfrican Program for the Control of Epizootics (PACE), organized a four-week (first-leg) intensive GIS training for five Nigerian veterinarians at the Regional Centre for Training in Aerospace Surveys (RECTAS), Obafemi Awolowo University Ile-Ife, Nigeria in 2001. A comprehensive GIS-based National Animal Disease Information System (NADIS) was being developed for Nigeria as part of PACE [8].

The NADIS database, a 3 in 1 system - database, disease 
reporting and information includes an epidemio-surveillance network and the assessment of the status of the four major trans-boundary diseases in Nigeria (African swine fever, foot-and-mouth disease, Newcastle disease and Rinder pest). It also includes a nationwide avian influenza random survey for the purpose of facilitating trade - covering access to (international) market, protection of animals against exotic or new diseases, evaluation of disease control programs and protection of human health [8]. These new initiatives within scientific education must be intrinsically connected to the forever-changing world of the ICTs.

Indeed, the virtual world is developing at an incredible rate and poultry famers have to adapt quickly and efficiently to these changes. The practical poultry man will recognize the fact that measures for the control of disease cannot be limited to sanitation and the treatment of sick birds, but, in reality, include such important matters as the selection of healthy stock, intelligent feeding, proper housing, and other details essential to the successful management of poultry which can only be achieved using such advanced technologies.

In the recent years, the common practice for Nigerian poultry farms in monitoring the diseases of their chickens is by sending extension workers to monitor and provide assistance on a regular basis. This practice consume large resources and time, especially when the breeder partners are distributed in various areas. The reporting mechanism is often done manually, by collecting the monitoring sheet at the end of a given period which leads to slow data collection and processing and wrong handling when extreme cases happen (such as fast spread of disease).

In addition, the conventional monitoring mechanism also slows the pace of response during an epidemic as information cannot easily be disseminated or accessed. As a result of this, there is the need to take the right preventive measures to address poultry diseases in Nigeria. This study presents the implementation of a web-based system for monitoring poultry disease from poultry farms remotely thus assisting government agencies and other stakeholders in poultry farming and management to track and monitor chicken diseases especially Newcastle disease and hence the necessary preventive and control measures to save their livestock and their livelihood income which provides economic benefits to them.

\section{Related Works}

Currently, many public health agencies use web-based data streams to monitor infectious disease outbreaks [17, 18]. Such systems have proved to be efficient in detecting outbreaks early and with reduced cost and increased transparency [19].

Several internet-scanning systems have been developed starting in 1997 with the Global Public Health Intelligence Network - GPHIN [12], and followed by many others such as the World Health Organizations of the Global Outbreak Alert and Response Network - WHOGOARN [18], the Program for Monitoring Emerging Diseases from the
International Society for Infectious Diseases - ProMED-mail [20], the Health Map system [21], and the Global Health Monitor based on the Bio Caster ontology [22].

More recently, internet search-term technologies such as Google and Yahoo (Polgreen et al., 2008) have been shown to have much potential for disease surveillance, but the information they recover is usually not detailed or reliable enough to estimate relevant epidemiological parameters of incipient outbreaks.

A system was developed in Indonesia which is aimed at helping Poultry farmers to monitor the impact of poultry diseases on chicken growth [23]. The system substitutes the role of poultry field instructor staff to collect data regarding the chicken growth and disease. It is expected that the system can provide more reliable data because the data is entered on daily based. However, the system was able to monitor the growth of broiler and also provide other effects of diseases on the broiler. A GIS-based system for monitoring environmental health by making it possible for environmental health workers to capture environmental health situation of any house in Nigeria real time while on the field was developed by [10]. Although the research identified some environmental factors that cause diseases, it focuses more on human health. Joami et al. (2008) developed a surveillance system for survey of Avian Influenza in Nigeria; the system was aimed at examining and monitoring Avian Influenza outbreak in Nigeria. The system was able to survey the disease but not easily accessible by local poultry farmers.

\section{Materials and Methods}

The researcher adopted the descriptive and exploratory designs that allowed the collection of data from sub-set of a poultry population whose analyses was generalized on poultry farms in Nigeria. The adoption of these methods gave a multi-faceted articulation on the different aspects of the study. A number of poultry farms were observed in order to assess their conditions and problems. In addition, data on Newcastle Disease was collected from sampled poultry farms through structured and unstructured personal interview. The data collected from this approach was used as a guide in order to develop the Web based monitoring system for poultry disease (WMSPD).

A. Requirement Analysis

The research required the identification of all system users and the data needed for managing poultry farms and the necessary activities carried out by users were identified. Also, the factors affecting poultry diseases and their corresponding influence were identified using interviews with poultry farm managers. Following the identification of the information necessary for managing poultry farms and the problems associated with the poultry farms the system requirements were explicitly identified in order to formulate a solution for the web-based development system for poultry disease management.

The design of the user requirements of this system was 
done using the Unified Modeling Language tools (UML). The users of the system were identified by the poultry managers interviewed and were selected based on the type of information either accessed or benefitting to the users, they are:

a System administrator - is responsible for managing the use of the monitoring system and also register every authorized user of the system;

b Poultry farmers - are primary users who provide information about state of poultry farms, they work within the poultry and provide information on observations made on poultry;

c Extension workers - are primary users who provide information about the state of poultry farms, they monitor poultry farms and give poultry managers feedback;

d Healthcare officers - provide information necessary for understanding poultry diseases into National databases;

e Government and non-governmental agencies - view the state of environmental health condition of poultry farms located at varying locations and thus channel any health-related assistance to the appropriate poultry farms; and

$f$ Disease control officers - can view the condition of poultry farms and determine the likelihood of any poultry related diseases based on observation made.

Following the user specification of the proposed webbased monitoring system for poultry disease in Nigeria poultry farms, the functional and non-functional requirements of the system was captured. This information was elicited as a result of ensuring that all necessary requirements of the system were captured during the requirements analysis. The functional requirements captured the intended behaviour of the system thus specifying the functions that the system components must be able to perform.

The system was expected to allow users to conveniently access the system from any remote location using the Internet and able to allow users concurrently provide and access information without causing neither failure nor reduced response time. The non-functional requirements were intended in to order to judge the operation of the system. Thus, the must be able to control and restrict unauthorized access by unprivileged users of the system. This system must be able to ensure the security of information with the use of encryption and access using passwords.

B. System Design

The Unified Modeling Language (UML) is the primary modeling language used to analyze, specify, and design software systems. From the late 1980s and well into the 1990s, numerous methodologies arose and were subsequently modified and refined. Many of these were strong in certain areas, weaker in others. This gave rise to methodologists adopting useful facets from other methodologies into their own. UML allows software models to be constructed, viewed and manipulated during analysis and design. A use-case is a type of UML diagram that identifies the actors (users) involved in an interaction and the names and the types of the interactions involved.

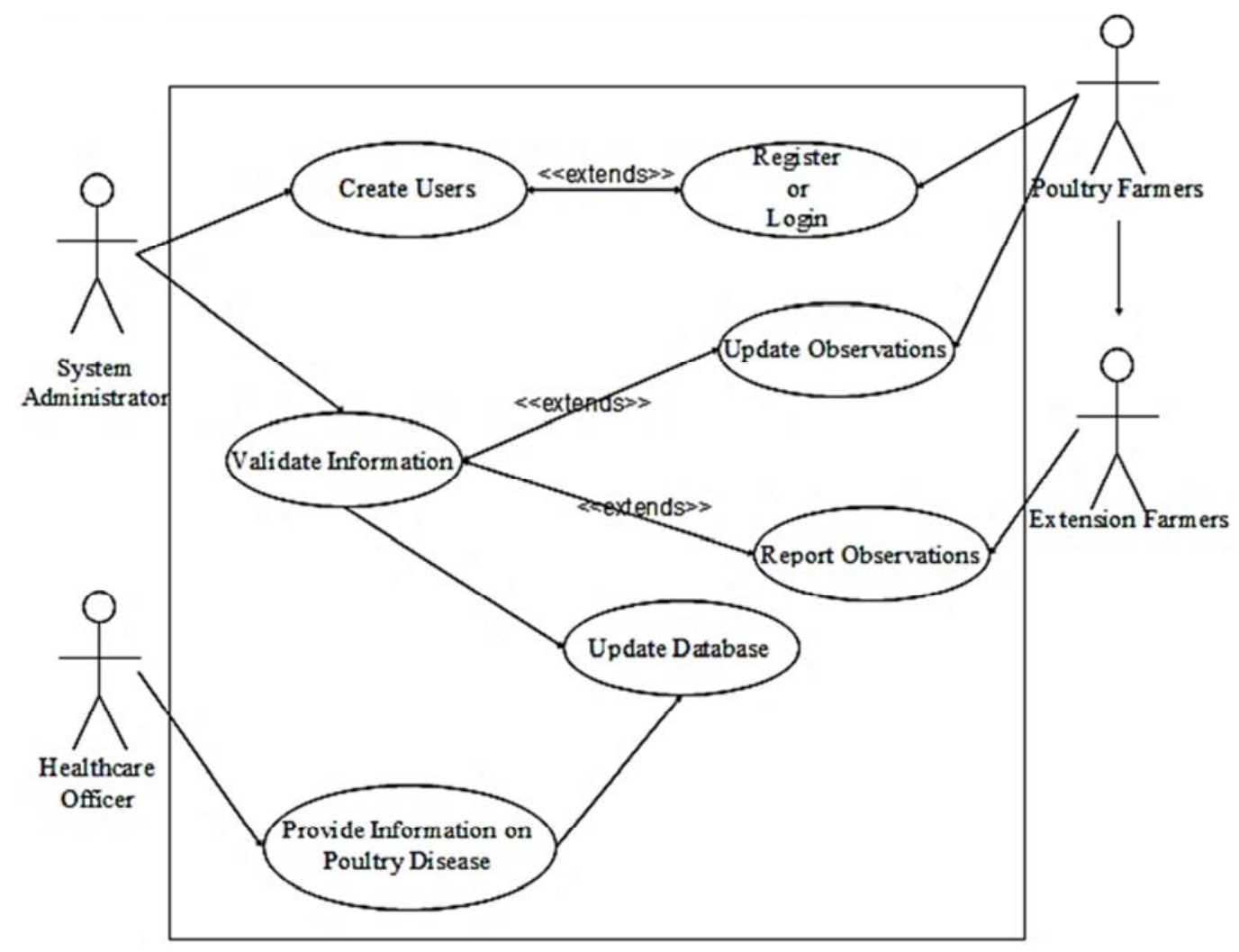

Figure 1. Data Insertion Use Case. 
The use case diagrams showed how data was entered and viewed from the proposed Poultry disease monitoring system. The first use-case describes data insertion Use-Case of the System for poultry disease monitoring system which involves the function of each actors of the system. The second use-case describes the data query section of the system which describes the functionalities that allow the viewing, updating, and searching, editing and deleting old data from the system. In the data insertion section of the developed poultry disease monitoring system, the poultry farmers/extension workers would be able to input information pertaining to a particular poultry into the system (Figure 1).

Information such as poultry disease, outbreak, date, cause, and casualty would be typed into the monitoring system. Also, the disease control officers would be able to enter all poultry disease data of the entire poultries in the specified location into the National Animal Disease Information System (NADIS) or national public health database.

The online monitoring and tracking of poultry disease status of all locations in Nigeria would also be possible. As a result, the proposed system is capable of monitoring diseases that are paramount in a particular area. In the data query usecase, Policy makers are able to view the environmental health state of any poultry farm by location in the study area. International organizations and non-governmental organizations will also be able to view the state of environmental health through a status report generated by the poultry farm's monitoring system from any location in the study area and channel any health assistance to the appropriate location (Figure 2).

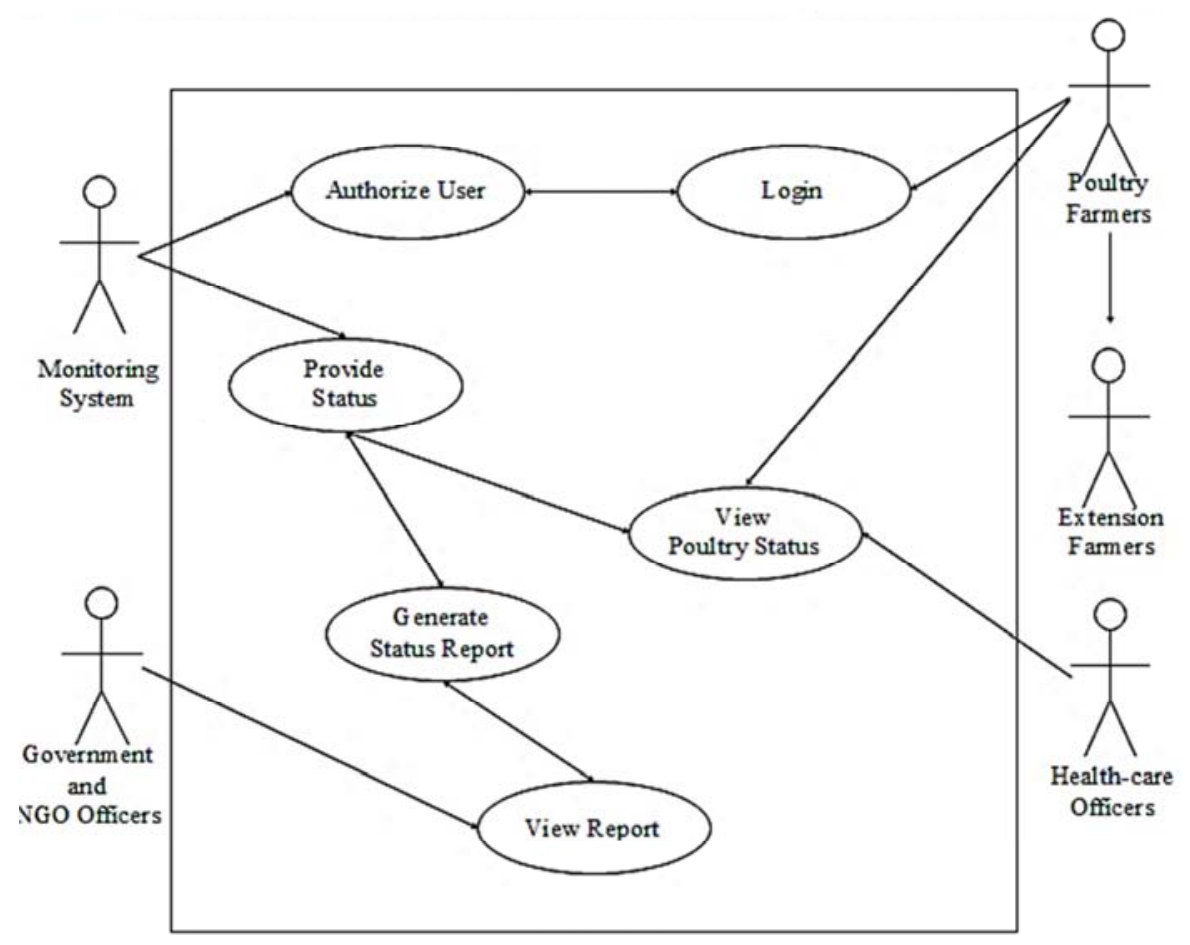

Figure 2. Data Query Use Case.

\section{System Architecture}

In the design of any web based related system, the use of system architecture is highly important. In the poultry disease monitoring system, there is a need to make the design of the system easily accessible, flexible and portable as much as possible. This was needful in order to allow users to easily make use of internet enabled mobile phones, tablets and other mobile devices, laptops, and desktop computers with web browsers to access the developed system. In this research, client-server system architecture was used and it is thin client-server. The Poultry disease monitoring system has two components namely: the server-side that runs on a server and a client-side that runs on the browser (Figure 3).

The system was modeled using the thin client-server architecture which makes use of the Web browser as the client. This architecture makes it possible to use any internetenabled device with a web browser installed to access the system; this is to ensure that the system is easily accessible, available and yet cheap to implement. In the client approach almost all the processing work was done on demand at the server-end while the task of the client-side was to display data and information to the user on the screen of the display monitor. This architecture was used due to its flexibility and easy accessibility. The users would not be required to install any software on their personal or mobile computers except a standard web browser, which is usually installed on the operating systems of personal and mobile computers provided by manufacturers. 


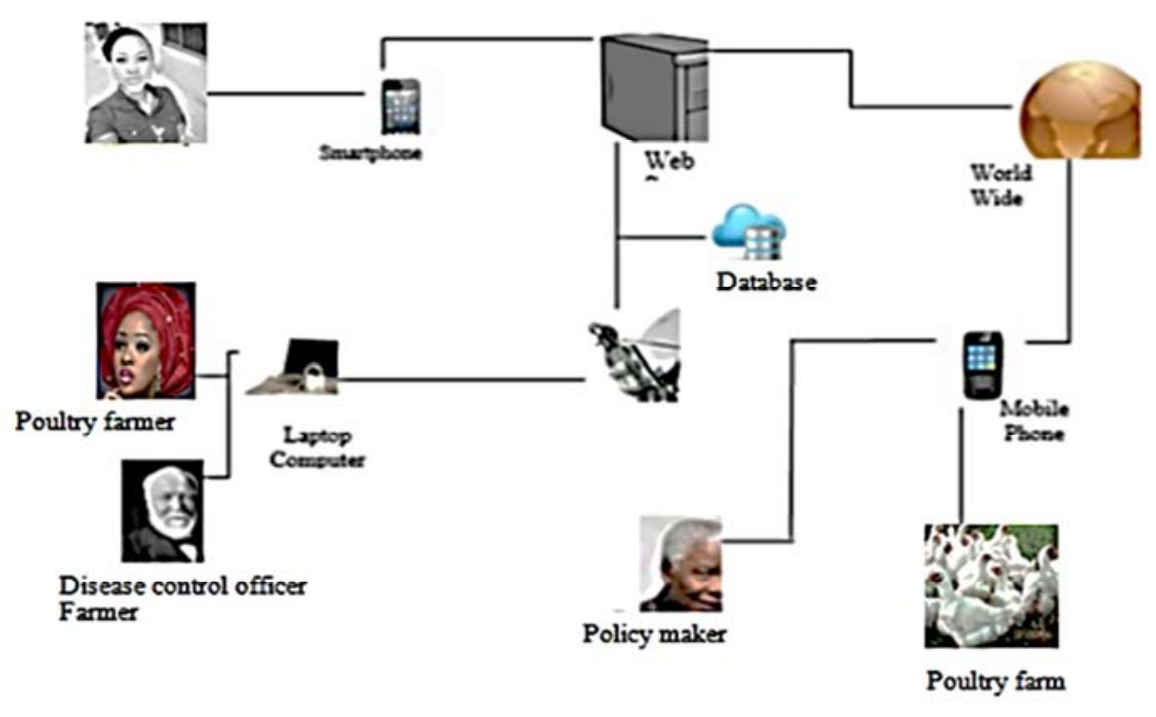

Figure 3. System architecture for poultry disease monitoring system.

The web based monitoring system for poultry disease supports a database, business logic and user interface. The User Machine (computer, laptop or a mobile/smartphone) is used to access the pages and forms of the web application. The Web Server allows the application to run and behave as though it is hosted on the Internet, using the WAMP Apache server. The back-end which consists of the web server and the database management system (DBMS) holds and manages the data pool used by the application. The PHP Script controls the exchange of data between the front-end and the application back-end. Clients do not require any powerful computer systems; users can use any computer with a web browser such as laptop/notebook, mobile phone, and desktop computer systems but servers require higher configuration (in terms of hardware) computer system since it is regularly subjected to heavy load; the servers are the HTTP and database server.

Using the monitoring system, a poultry farmer on the field, upon noticing or suspecting poultry disease will alert the system manager who immediately will relay the information to the relevant disease control authorities so that immediate action could be taken. Also, other stakeholders can play a very important role as they can also report an outbreak by submitting reports on the monitoring system for poultry disease Website. Finally, based on the information available in the system, all stakeholders especially the policy makers, who usually are government officials, can put in place relevant preventive and control measures to prevent or manage an outbreak.

\section{System Implementation}

In order to develop the prototype, the poultry disease monitoring database was developed using MySQL. In the process of developing the poultry disease database, different tables, files, records and fields were created. As a result, different poultry condition factors such as the sanitary condition, the date of the disease outbreak, description of the birds involved, the cause of the incidence, disease type and effects, poultry size and location, control measures, and livestock status were stored in the database.

The user interface which allows the user to navigate the system and also facilitate interaction with the database was also implemented using the Windows Interface Menu (WIMP) which makes the system simple and user-friendly. Therefore, the poultry disease monitoring information system was implemented in design to accommodate users with varying skills and competence in the area of computer usage. The system's interface was implemented using Adobe Dreamweaver; while Apache was the web server used to provide the basic functionality of the monitoring system and PHP was used as a scripting language to program the serverside manipulation of the knowledge in the database. A well designed and simple user interface provides users with a better understanding of the system behaviour and overall functionality.

\section{Results and Discussions}

Following the implementation of the system, the results of the functionality of the proposed poultry disease monitoring system are presented using the interfaces used to implement important parts of the system. The user interface allows the user to navigate the proposed system and communicate with the database. The system design is simple enough for use by anyone with little knowledge of computing and since the targeted end users are poultry farmers and disease control department of local government area in the study area, the system is user friendly and made easy to use through the use of windows and interface menu. The interfaces built into the system include the:
a Home page;
b Registration Page;
c Login Page; 
d Disease statistics and report page;

e Disease report transfer page;

f The Admin Dashboard Page; and

$\mathrm{g}$ Poultry disease distribution page.

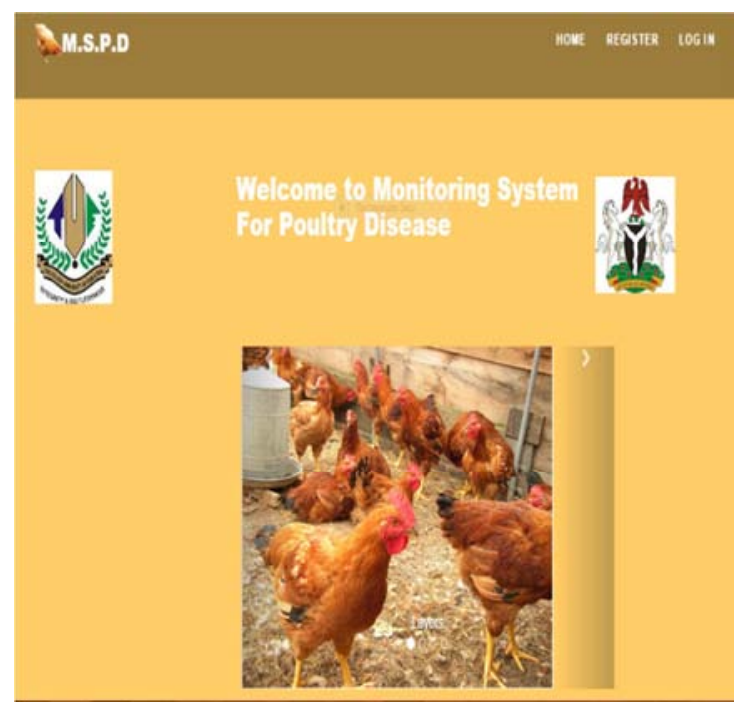

Figure 4. Home page of monitoring system for poultry disease.
The implemented system's index page is the home page that is used to navigate to different parts of the monitoring system by users accessing the system. This index page contains links to help existing users of the Monitoring System for Poultry Disease (MSPD) to log in and new users to register (Figure 4). There is also news relating to poultry disease prevention on the homepage. On the homepage, the user is able to submit disease report, new users are able to register and returning users are able to login. Users can easily access this interface by logging to the unified resource locator of the monitoring system but can only be able to perform other actions only if the person is authorized and has an account with the monitoring system.

Following access to the homepage, new users need to have registered to the system following which username and passwords are provided by the system administrator as long as such users have been verified as authorized users. On the Registration page, the intended user of the system is required to provide his/her username, password, phone number, Poultry Name, Location, e-mail address. Upon entering these details, the data would be sent into the database. All fields are required for successful registration and if one field is left empty, a prompt will come up (Figure 5).

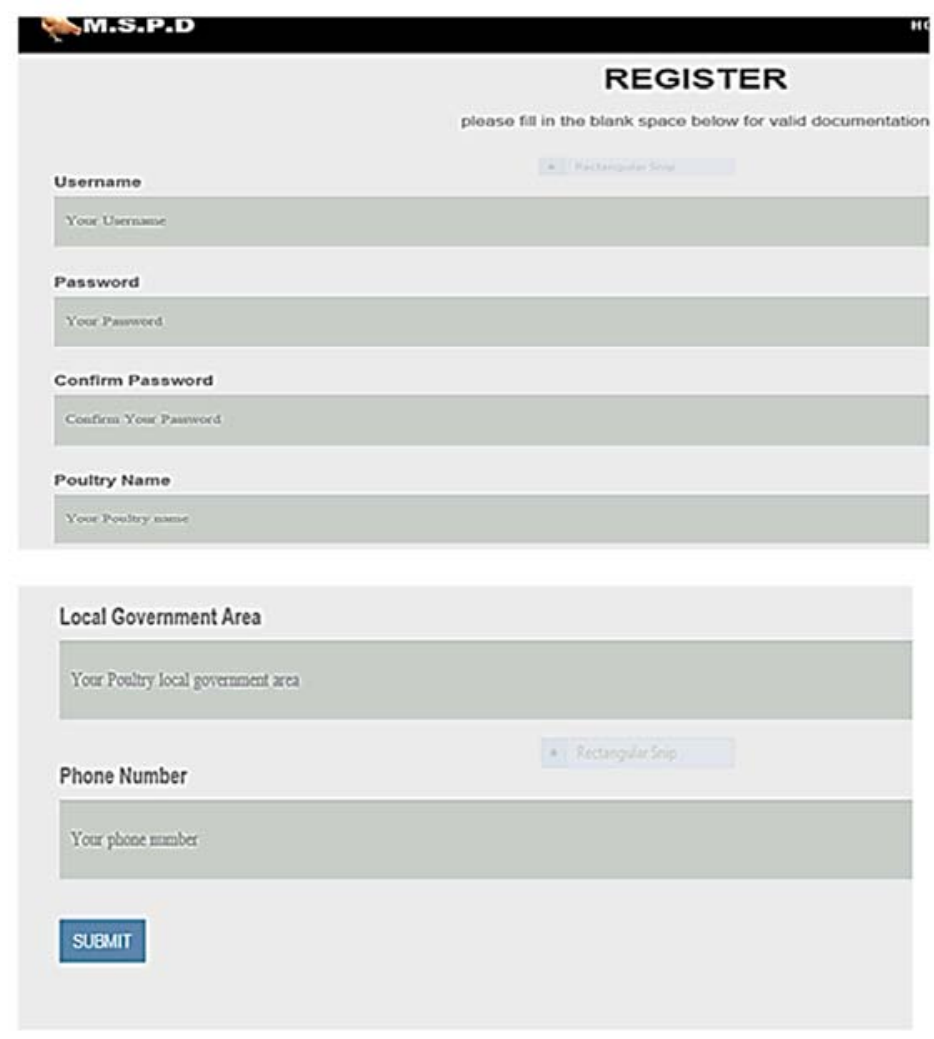

Figure 5. Registration page for new system users.

If users accessing the system from the home page are already registered to the system to by the system administrator, they can access the system by providing the username and password provided by the administrator at the point of verifying their registration to the system using the login interface (Figure 6). When the user of the system encounters the login page, he is required to enter his username and his password to be able to log into the system. For a login attempt to be successful, the username and password combination input by the user must correspond to values that are available in the database. 


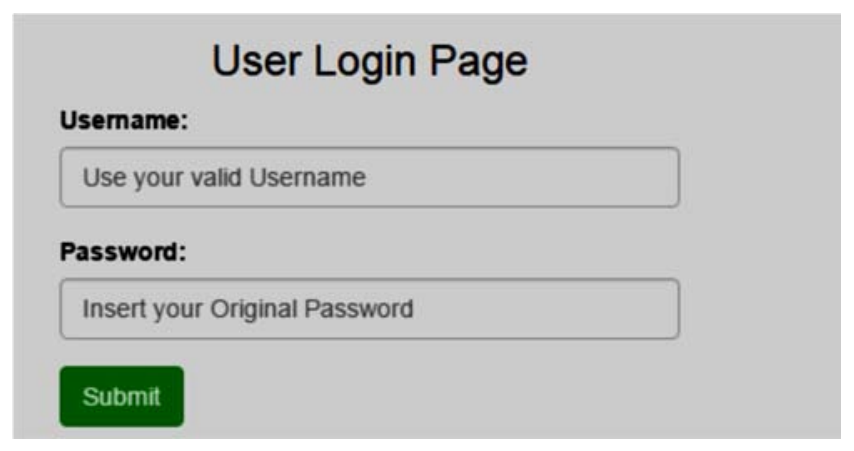

Figure 6. Login dialog box.
The disease report transfer page is used to produce information regarding poultry diseases occurring within a registered poultry farm in the system's database (Figure 7). Such diseases reports can then be forwarded (transferred) to the necessary and appropriate health department for the necessary actions to be taken regarding the disease. This is to ensure that the requirements necessary for prompt response by healthcare officials to any observed poultry disease is responded to. The page records information regarding the name of the poultry, the poultry address, notification form, where the information needs to be transferred to and the reason for the transfer. The result of this page is then presented as a report to be viewed by interested stakeholders.

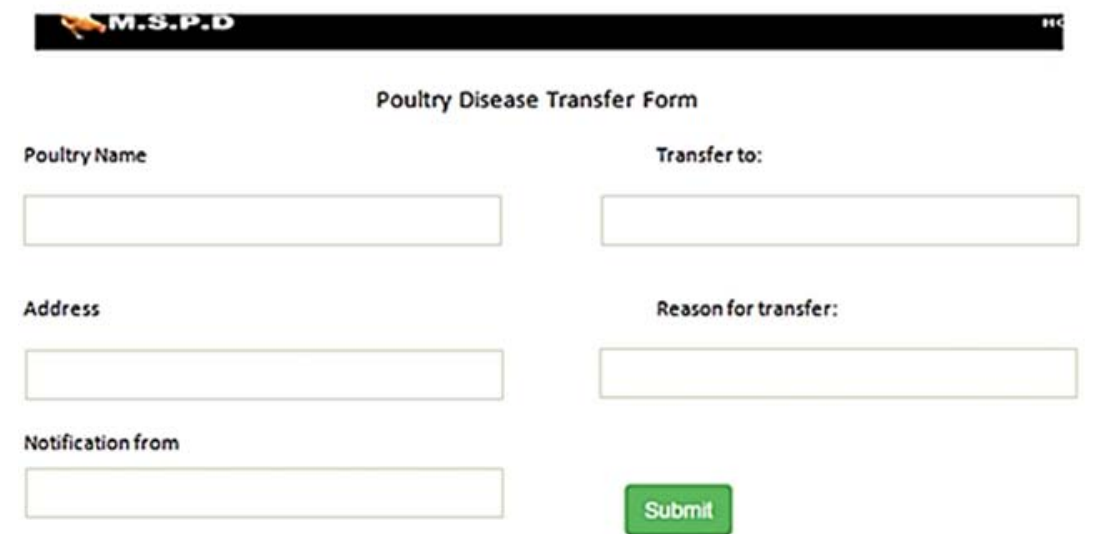

Figure 7. Poultry disease transfer page.

The process of reporting a poultry disease using the poultry disease transfer page generates a report which can be accessed the health-care officers in order to understand the state of poultry farms and the observations made regarding the onset of a poultry disease (Figure 8). The reports stores and provides information about the state of a poultry farm including the address of the poultry farm, local government and state. Also, the report provides information on the contact number of the representative of the poultry farm responsible for providing reports. In addition a disease report section is used to provide details on the state of the farm and the observations made on the poultry, such information is then useful to the stakeholders in providing decision support to policy makers and other important government agencies.

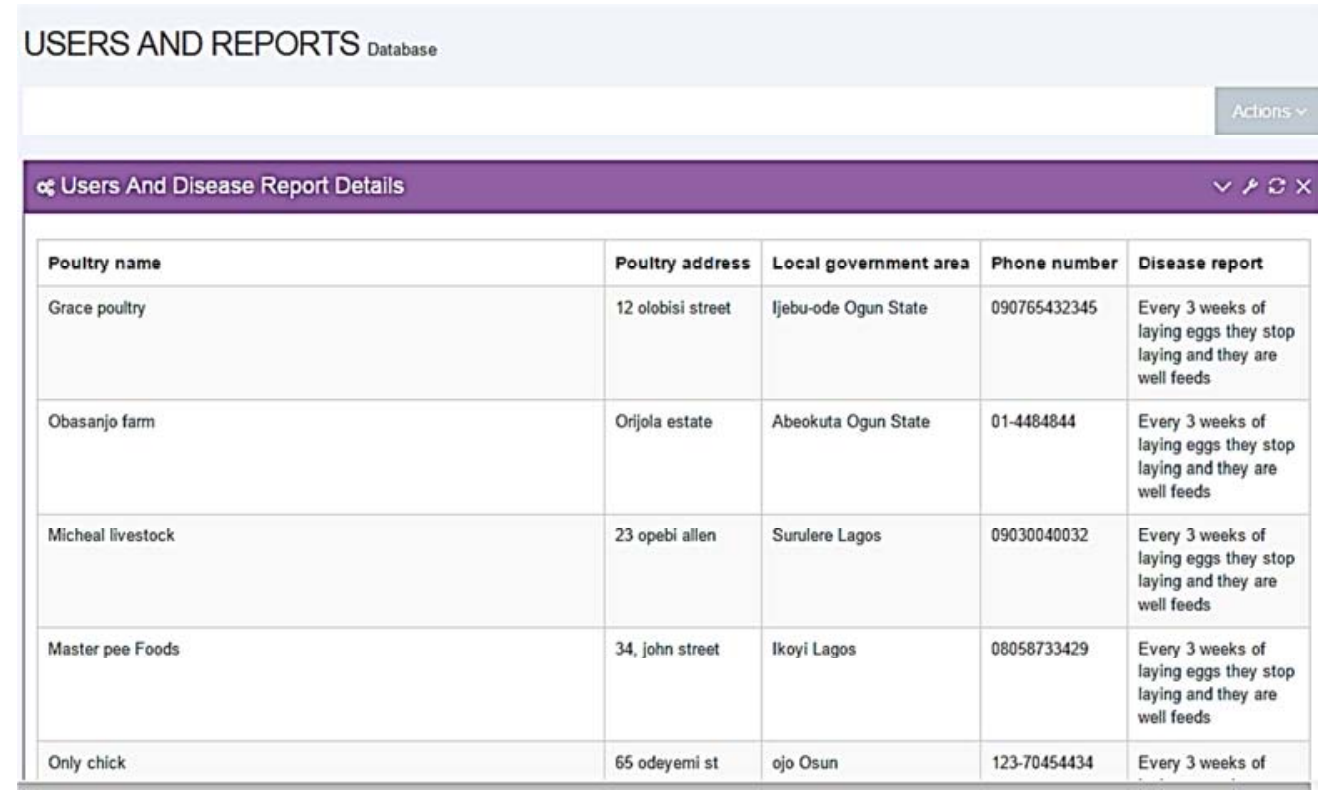

Figure 8. A section of the disease statistics report page. 
There is also an administrator's dashboard that performs the administrative functions such as modifying site pages, adding events, and customizing the contact database. Administrator can log in and access the admin backend, an area reserved for site and account administration, and not seen by ordinary members or visitors to your site. The administrator have been granted the technical ability to perform certain special actions which include the ability to block and unblock user accounts from editing, edit fully protected pages, protect and unprotect pages from editing, delete and undelete pages, rename pages without restriction, and use certain other tools (Figure 9).

\section{Admin Panel

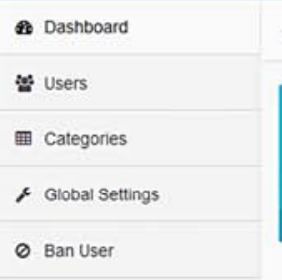

Dashboard it all starts here
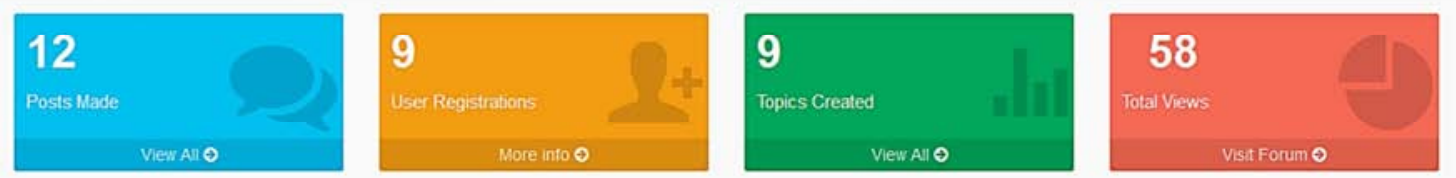

Figure 9. Administrator's dashboard.

There are also additional interfaces required for viewing the distribution of information about reports coming from poultry farm according to their spatial distribution on a map. This was achieved with the use of Google Map Application Programming Interface (API) which provided access to the functionalities of the Google Map software so that the graphical location resources were utilized for the purpose of showing the distribution of reports coming from poultry farms. This interface displays the various states in south-western Nigeria showing the record of poultry farms that have any infected poultry reported (Figure 10). The poultry disease distribution module can only be functional or available as long as there is internet connection on the device being used to access the system and can be authorized by all users. The distribution of poultry infection can also be observed spatially within a state as shown in Figure 11.

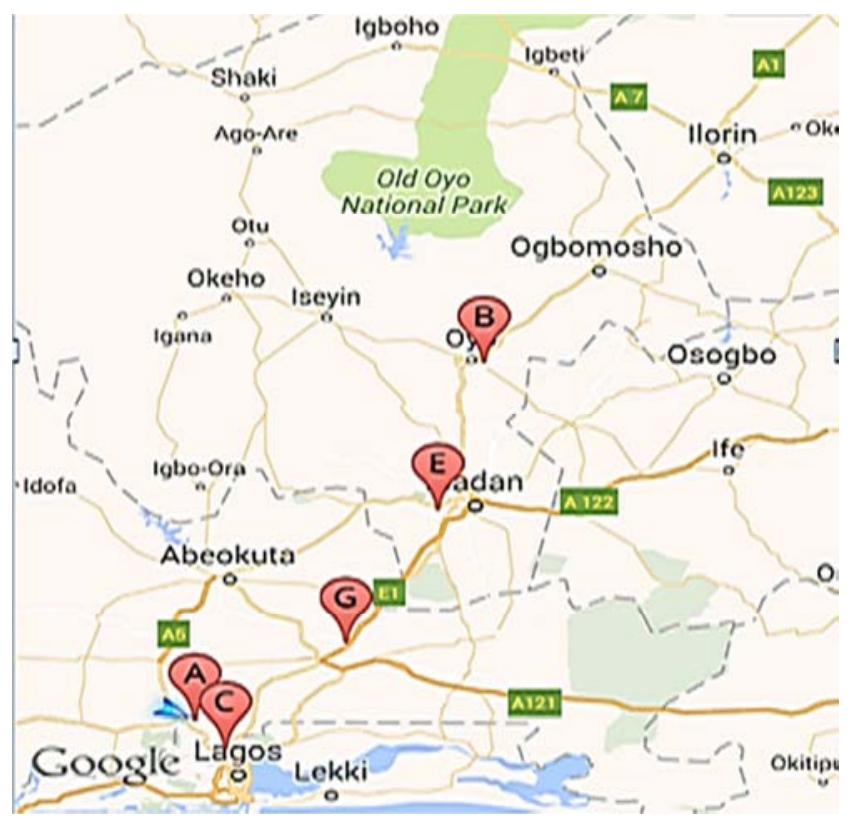

Figure 10. Poultry disease distribution in south-west Nigeria.

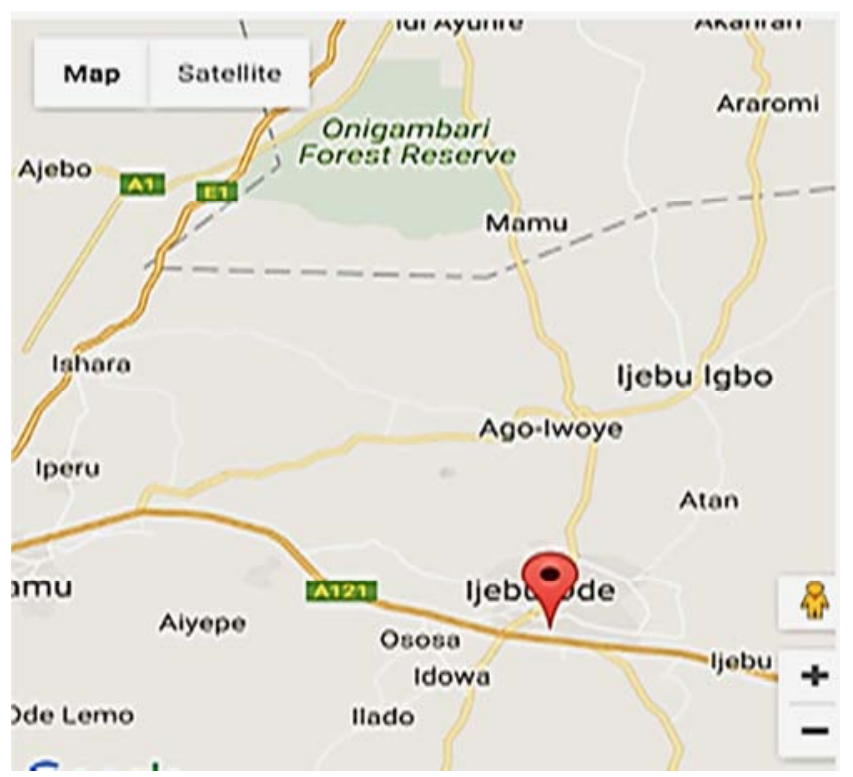

Figure 11. Poultry disease distribution in Ijebu-Ode, Ogun State.

The proposed monitoring system for poultry disease was developed to facilitate the easy storage and monitoring of disease related information which can be easily accessed by Admin, poultry farmers and health care Officers who are registered to the system as authorized users. This system would also act as a means of providing effective and timely information concerning the state of poultry diseases in Nigeria, given the information found therein. The system also allows users to query information from different locations in South-western Nigeria. This system will be very useful to Nigeria as a result of the kind of information that is stored in the system.

The poultry disease monitoring system was developed using the adopted data model to gather information comprehensively about poultry diseases which help to monitor and control the widespread of the disease and provide a database for poultries in south-western Nigerian. The system will help determine the distribution rate of 
poultry disease in south-western Nigeria and its prevalence rate in this region of the country. The system allows the users to register information about poultry disease, view disease statistics and disease distribution in different states within south-west Nigeria. Through the poultry disease monitoring system, the system administrators, managers and maintenance officers can be able to access the information available in the system as long as they are registered. The information provided to the users can be helpful in simplifying fresh policies that can help improve the state of poultry disease in Nigerian at large. In overall, this system can become a central repository to all health across Nigeria with all information relating to poultry disease and health stored in one single system where analysis can be made to identify the kind of relationship that exist among the data stored. This system will hopefully aid effective and efficient intervention in the rate at which poultry diseases is affecting the health and production of poultry birds in Nigerian.

\section{Conclusion}

In Nigeria it is estimated that poultry supplies about $10 \%$ of the total meat needs and out of about 150 million poultry birds, 102 million are indigenous. The indigenous village chicken is identified as a way of providing rural women with diverse income earnings and employment. However, the endemic Newcastle and associated mortalities constitute a major obstacle to the promotion of large holdings of these birds. The disease is worldwide in distribution and virus strains of wide varying degrees of pathogenicity exist.

The design and development of a web-based monitoring system that could help to monitor, prevent and control the poultry disease in Nigeria is proposed in this research. The system can assist poultry farmers and stakeholders to monitor disease outbreak in order to reduce the rate of poultry mortality. The system can alert the respective state animal husbandry departments for preventive actions against an outbreak of poultry related diseases. The system consists of two main parts, the data collection module and monitoring module. The data collection system facilitates the short message service and mobile application. Mobile application is selected because many poultry farmers uses mobile device intensively. However, because the poultry farms are mostly located in remote areas, which sometimes are not covered with data network; therefore it is not effective to use only mobile application. For this reason, short message service is selected to solve the drawback of mobile application. The system substitutes the role of poultry field instructor staff to collect data regarding the poultry disease. It is expected that the system can provide more reliable data because the data is entered on daily basis.

The system will help the farmers to save their livestock and their livelihood income which provides economic benefits to them. With the web based monitoring system for poultry disease, Users can create new records in poultry diseases, edit existing records or search specific records based on the location. Any authorized user would be able to go online and view poultry condition of any desired location. Users also can predict likelihood of poultry disease in any location online. This system demonstrates that web based system may enhance poultry production in two ways. One is to enhance tracking and monitoring data management and this is achieved by editing, reporting, deleting poultry disease data. The other is to enhance tracking information services, such as the provision of a query, generation of reports, among others.

\section{References}

[1] Kamani, J., et al., Tetrameres fissispina Infection in Local Chickens in Taraba State, Nigeria, Case Report. International Journal of Poultry Science, 2008. 7 (12): p. 1216-1218.

[2] Iqbal, S. and Z. Pampori, Production potential and qualitative traits of indigenous chicken of Kashmir. Livestock Research for Rural Development, 2008. 20 (11): p. 14.

[3] Bawa, G., et al., Farmers' perception of the avian influenza (birdflu) epidemic in some parts of Northern Nigeria. American-Eurasian Journal of Scientific Research, 2010. 5 (3): p. 170-175.

[4] Anjum, A., S. Hassan, and G. Arbi, Infectious bursal disease in chickens in Pakistan. Pakistan Veterinary Journal, 1993. 13: p. 54-54.

[5] Lambert, C. and A. Kabar, Egg drop syndrome (EDS 76) in New Caledonia, first diagnosis and control. Revue d'Élevage et de Médecine Vétérinaire de Nouvelle Calédonie, 1994 (18): p. 7-14.

[6] Sa'idu, L., et al., Retrospective study of Newcastle disease cases in Zaria, Nigeria. Nigerian Veterinary Journal, 2006. 27 (3): p. 53-62.

[7] Kraneveld, F., A poultry disease in the Dutch East Indies. Ned Indisch Bl Diergeneeskd, 1926. 38: p. 448-450.

[8] Babalobi, O. O., The challenge of changing trends in the development and use of information and internet web communication technologies for veterinary medicine education in Nigeria. 2010.

[9] Simoes, J., Information communication technology applied to veterinary education in early XXI century. Veterinaria. com. pt. 2010; 3 (1): 1-29.

[10] Idowu, A. P., E. R. Adagunodo, and O. A. Esimai, Development of a web based environmental health tracking system for Nigeria. International Journal of Information Technology and Computer Science (IJITCS), 2012. 4 (7): p. 61.

[11] Chretien, J.-P., et al., Syndromic surveillance: adapting innovations to developing settings. PLoS Med, 2008. 5 (3): p. e72.

[12] Mykhalovskiy, E. and L. Weir, The Global Public Health Intelligence Network and early warning outbreak detection: a Canadian contribution to global public health. Canadian Journal of Public Health/Revue Canadienne de Sante'e Publique, 2006: p. 42-44. 
[13] Yang, C., et al., Use of mobile phones in an emergency reporting system for infectious disease surveillance after the Sichuan earthquake in China. Bulletin of the World Health Organization, 2009. 87 (8): p. 619-623.

[14] Buehler, J. W., et al., Syndromic surveillance and bioterrorism-related epidemics. Emerging infectious diseases, 2003. 9 (10): p. 1197-1204.

[15] Sugawara, T., et al., An experimental study for syndromic surveillance using OTC sales. Kansenshogaku zasshi. The Journal of the Japanese Association for Infectious Diseases, 2007. 81 (3): p. 235-241.

[16] Babalobi, O. and P. Cowen. PROMED-mail: An electronic mail disease-reporting forum. in Proceedings of the 38th National Congress of the Nigerian Veterinary Medical Association (NVMA), Topo, Badagry, Lagos. 2001.

[17] Grein, T. W., et al., Rumors of disease in the global village: outbreak verification. Emerging infectious diseases, 2000. 6 (2): p. 97.

[18] Heymann, D. L. and G. R. Rodier, Hot spots in a wired world: WHO surveillance of emerging and re-emerging infectious diseases. The Lancet infectious diseases, 2001. 1 (5): p. 345353.

[19] Wilson, K., B. von Tigerstrom, and C. McDougall, Protecting global health security through the International Health Regulations: requirements and challenges. Canadian Medical Association Journal, 2008. 179 (1): p. 44-48.

[20] Woodall, J. P., Global surveillance of emerging diseases: the ProMED-mail perspective. Cadernos de Saúde Pública, 2001. 17: p. S147-S154.

[21] Brownstein, J. S., et al., Surveillance Sans Frontieres: Internet-based emerging infectious disease intelligence and the HealthMap project. PLoS Med, 2008.5 (7): p. e151.

[22] Collier, N., et al., BioCaster: detecting public health rumors with a Web-based text mining system. Bioinformatics, 2008. 24 (24): p. 2940-2941.

[23] Martindah, E., N. Ilham, and E. Basuno, Biosecurity level of poultry production cluster (PPC) in West Java, Indonesia. International Journal of Poultry Science, 2014. 13 (7): p. 408415 . 